\title{
Non-invasive measurement of adrenocortical activity in a myrmecophageous mammal, the bat-eared fox
}

\author{
Aliza le Roux ${ }^{1^{*}}$, Helene Botha ${ }^{2}$, Fredrik Dalerum ${ }^{3-5}$, Andre Ganswindt ${ }^{4,6,7}$ \\ 1) Zoology and Entomology, University of the Free State, Qwaqwa campus, Private Bag X13, \\ Phuthaditjhaba, 9866, South Africa \\ 2) Zoology and Entomology, University of the Free State, PO Box 339, Bloemfontein, 9300, \\ South Africa \\ ${ }^{3)}$ Research Unit of Biodiversity (UO, CSIC, PA), University of Oviedo, Mieres Campus, 33600 \\ Mieres, Spain \\ 4) Mammal Research Institute, Department of Zoology and Entomology, University of Pretoria, \\ 0028, Pretoria, South Africa \\ 5) Department of Zoology, Stockholm University, 106 91, Stockholm, Sweden \\ 6) Endocrine Research Laboratory, Department of Anatomy and Physiology, University of \\ Pretoria, 0110, Onderstepoort, South Africa \\ ${ }^{7}$ ) National Zoological Gardens of South Africa, 0001, Pretoria, South Africa \\ * Corresponding author: Email: lerouxa3@qwa.ufs.ac.za, Tel: +27 58718 5327, Fax: +27 58 \\ 7185034
}

\section{Abstract}

Measuring physiological stress reactions through the quantification of plasma cortisol often involves physically restraint, which acts as a stressor itself. Here, we present the validation of a non-invasive method for assessing adrenocortical activity as an indicator of stress in the bat-eared fox (Otocyon megalotis). By conducting an adrenocorticotropic hormone (ACTH) challenge, we examined the suitability of three enzyme immunoassays (EIAs) detecting 11,17 dioxoandrostanes (11,17-DOA) as well as faecal glucocorticoid metabolites (fGCM) with a $5 \beta$-3a-ol-11-one $(3 \alpha, 110 \times 0-C M)$, or $11,17,21$ trihydroxy-4-ene-20-one structure (cortisol), respectively, for monitoring stress-related physiological responses in male and female bat-eared foxes. Our results suggest that the cortisol EIA seems most suitable for measuring fGCMs in this myrmecophageous mammal. Using the cortisol EIA, we compared fGCM concentrations of three populations of foxes. Only one 
population experienced a sudden change in social environment and the assay appeared to effectively detect the resulting increase in stress hormone levels. Therefore, the identified EIA is effective at detecting intraspecific variation in fGCM levels, and hence a useful tool to evaluate physiological stress responses in this species.

Keywords: adrenocorticotropic hormone challenge, faecal glucocorticoid metabolites, non-invasive hormone measurement, Otocyon megalotis, stress

\section{Introduction}

Recent years have seen an upsurge in non-invasive methods to measure physiological stress reactions, especially in wild mammals (Palme et al. 2005; Hodges et al. 2010; Ganswindt, Brown, et al. 2012). The array of available methods has been reviewed elsewhere (e.g., Palme et al. 2005; Sheriff et al. 2011; Cook 2012), but generally rest on the principle that circulating levels of glucocorticoids can be evaluated through a parameter of adrenocortical activity, such as the glucocorticoid metabolites found in urine or faeces. A technique that shows particular usefulness in wild mammals is measuring glucocorticoid metabolites in faeces - a matrix that can be collected more readily than urine or saliva. Since the presence and relative abundance of faecal glucocorticoid metabolites (fGCM) depends greatly on species-specific diet, rates of digestion, sex differences, and gut-passage times, this method has to be carefully validated for each species in which its use is deemed necessary (Touma and Palme 2005; Goymann 2012).

Here, we investigate the suitability of using $\mathrm{FGCM}$ measurement to assess stressors in the bat-eared fox, Otocyon megalotis, a medium-sized insectivorous mammal widely distributed across southern and east Africa (Clark 2005). Bat-eared foxes are pair-living, social canids that are typically housed in pairs or family groups when in captivity. Nutritional stress during lactation appears to be a fundamental reason for this species' unusually high reliance on paternal care (Pauw 2000), yet no study has yet measured physiological stress reactions in bat-eared foxes. Our aims with this investigation were therefore to (1) physiologically validate different EIAs for 
measuring fGCM to be able to select the best-suited assay for non-invasive monitoring of adrenocortical activity in this species, and (2) test whether different populations of foxes (where one population experienced a recent change in social housing conditions) show differences in their fGCM levels.

\section{Methods}

\section{Study population}

The test subjects for this study - all adult foxes - were drawn from three separate locations: a captive pair of bat-eared foxes at the National Zoological

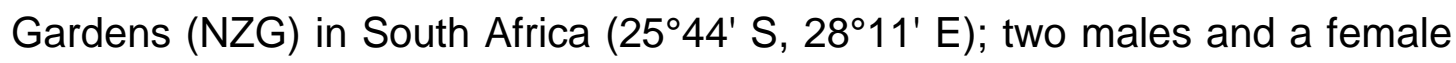
from a privately owned wildlife sanctuary in Kestell (My Little Africa (MLA): $28^{\circ} 18^{\prime} \mathrm{S}, 28^{\circ} 42^{\prime} \mathrm{E}$ ); and four individuals (three male, one female) from a wild population in the Benfontein Nature Reserve (BEN), Kimberley (28 $99^{\prime} S$ and $\left.24^{\circ} 81^{\prime} \mathrm{E}\right)$. The pair at the NZG was used in the initial identification of a reliable EIA, and was originally housed together in a $70 \mathrm{~m}^{2}$, readily divisible enclosure. Five days prior to a standard adrenocorticotropic hormone (ACTH) challenge test, the individuals were separated within their enclosure, maintaining visual and olfactory contact, while allowing individual collection of faecal samples. The ACTH challenge, described in the following section, followed well-described methods (Hulsman et al. 2011; Conforti et al. 2012; Ganswindt, Tordiffe, et al. 2012), and was performed to assess the suitability of various EIAs. After ACTH administration, the animals remained separate, housed individually in the quarantine enclosure at the NZG veterinary hospital until the end of the experiment. Visual and olfactory contact was maintained during this period. The study was conducted with ethical clearance from the University of the Free State (\#11/2013), the University of Pretoria (EC031-07), and the National Zoological Gardens (P12/16).

The second study population was a trio of captive foxes housed at the MLA sanctuary in a $30 \mathrm{~m}^{2}$ enclosure, with researchers having direct physical and visual access to the animals. These animals had been in captivity for several years, and were likely wild-caught. The MLA population was observed for a period of one month in order to collect faecal material. The third study population consisted of free-ranging bat-eared foxes occurring at the Benfontein Nature Reserve, an 11000 ha game farm consisting of dry Karoo, 
grassland and Kalahari savannah (Marneweck et al. 2015). This population has been under behavioural observation from November 2010, and faecal samples were opportunistically collected from animals habituated to the presence of an observer in a field vehicle (le Roux et al. 2014). After identifying the most suitable assay, we compared baseline fGCM levels of the NZG population (pre-ACTH data), MLA, and BEN, with the aim of determining whether or not the assay can detect the impact of the social and housing disruption that occurred at the NZG.

\section{ACTH challenge}

In the morning of the ACTH challenge, the two study animals at NZG were net-captured individually and anesthetized by using a combination of medetomidine $(0.05 \mathrm{mg} / \mathrm{kg})$ and ketamine $(5 \mathrm{mg} / \mathrm{kg})$. The female fox incurred an injury to her carpal joint ligaments during the capture procedure, which was immediately surgically corrected. For each individual, an initial blood sample was taken upon arrival at the on-site veterinary hospital about 30 minutes after capture. Subsequently each animal was injected intramuscularly with 10 IU of synthetic ACTH (Synacthen Depot, Novartis). A second blood sample was taken 40 minutes after ACTH administration. Directly after extraction, all blood samples were placed on ice for 60 minutes to allow clotting. Serum was obtained through centrifugation at $2350 \mathrm{G}$ for 5 minutes, transferred to glass tubes and frozen at $-20{ }^{\circ} \mathrm{C}$ until assayed. Anaesthesia was reversed with 0.2 mg Antisedan, after which the animals were kept individually in quarantine enclosures near the hospital. All faecal samples were collected and stored using the protocol described below for five days following the challenge, after which the study animals were released together into their original enclosure.

\section{Faecal sample collection and extraction}

At all three sites, faecal samples were only collected when the defecator could be identified, and the storage protocol was identical across sites. Prior to the ACTH challenge in the NZG set-up, faecal samples2 were collected during the day (09:00 to 16:00), within one hour of defecation. Enclosures could not be accessed during the night (16:00 to 09:00); therefore all faecal samples produced overnight were collected the following morning. This yielded 7 pre- 
ACTH samples from the female and 12 from the male. Animals at MLA were readily accessible. Therefore four samples from the single female, with five and six samples from the two males, were collected within five minutes of defecation, by day or night. At BEN, faecal samples were opportunistically collected from animals habituated to the presence of an observer in a field vehicle, yielding a total of three samples from one female, and two to five samples from three males. Thoroughly mixed aliquots of each faecal sample were immediately placed on ice and frozen within one hour after collection. Samples were stored at $-20^{\circ} \mathrm{C}$ until further processing at the Endocrine Research Laboratory at the University of Pretoria.

To determine the stability of fGCM post defecation, fresh faecal material was additionally collected from the NZG animals prior to the ACTH challenge. The collected material was thoroughly mixed and divided into 21 equal subsamples, which were stored at ambient temperature. Subsequently, 3 subsamples were frozen at $-20^{\circ} \mathrm{C}$ after $0,1,2,4,8,16$, and 24 hours post defecation, respectively.

The frozen faecal samples were lyophilized (Christ ${ }^{\circledR}$ Alpha 1-2LD plus, $\sim 1 \mathrm{mbar},-55^{\circ} \mathrm{C}$ ) and subsequently pulverized using a mesh strainer to remove undigested material (Fieß et al. 1999). Between $0.05-0.06 \mathrm{~g}$ of faecal powder was then extracted using $3 \mathrm{ml}$ of $80 \%$ ethanol in water. After vortexing for 15 minutes, the mixtures were centrifuged for 10 minutes at $1500 \mathrm{~g}$, and the supernatants transferred into microcentrifuge tubes and stored at $-20^{\circ} \mathrm{C}$ until steroid analysis.

\section{Steroid analysis}

Serum cortisol levels were determined by using a Coat-A-CountC Cortisol RIA (Siemens Medical Solutions Diagnostics) following the prescribed protocol (see also Ganswindt, Tordiffe, et al. 2012). Details regarding assay sensitivity, intra-assay coefficient of variation, and major cross-reactivities are mentioned in Ganswindt et al. (2012).

Faecal extracts were measured with EIAs detecting 11,17 dioxoandrostanes (11,17-DOA) as well as fGCMs with a $5 \beta$-3a-ol-11-one (3a,110xo-CM) or 11,17,21-trihydroxy-4-ene-20-one structure (cortisol), respectively. Details of the three EIAs, including cross-reactivities are 
described by Möstl and Palme (2002) for 3a,110xo-CM and by Palme \& Möstl (1997) for 11,17-DOA and cortisol. Sensitivity of the cortisol EIA was $1.6 \mathrm{ng} / \mathrm{g}$, and $3.3 \mathrm{ng} / \mathrm{g}$ for the other two group-specific assays. Serial dilutions of extracted faecal samples gave displacement curves that were parallel to the respective standard curves in all assays (difference in slope was < 1\% for $3 \alpha, 110 \times 0-C M,<3 \%$ for 11,17-DOA, and < 4\% for the cortisol EIA). Intra- and inter-assay coefficients of variation, determined by repeated measurements of high and low value quality controls ranged between $8.4 \%$ and $15.2 \%$ for cortisol, $1.9 \%$ and $13.9 \%$ for $11,17-\mathrm{DOA}$, and $6.1 \%$ and $15.5 \%$ for the 3a,110xo-CM EIA. Assays were performed on microtiter plates as described by Ganswindt et al. (2002).

\section{Data analysis}

Data from the three distinct assays were contrasted using the baseline data collected in the five days prior to the ACTH challenge. Similarly, overall preinjection individual median fGCM concentrations were used to determine postACTH increase in measured steroid concentration. This assay was subsequently applied to samples from the two other populations, contrasting the three sites using Kruskal-Wallis ANOVA. Only the pre-ACTH faecal data from NZG was used in this comparative analysis.

\section{Results}

\section{ACTH challenge}

In the male fox, serum cortisol concentration increased from $136 \mathrm{nmol} / \mathrm{l}$ to 144 $\mathrm{nmol} / \mathrm{l}$, while the female's cortisol levels changed from $121 \mathrm{nmol} / \mathrm{l}$ to 176 nmol/l. The 3a,110xo-CM EIA detected a 4-fold (female: Fig. 1a) and 5.5-fold (male: Fig. 1b) increase in fGCM concentrations above pre-injection levels. The 11,17-DOA assay revealed a 5-fold (female) and 7.5-fold (male) increase in fGCM levels above baseline concentrations, and the cortisol EIA detected a 90-fold (female) and 9-fold (male) increase in fGCM concentrations above pre-ACTH administration levels. Peak fGCM concentrations were found $20 \mathrm{~h}$ post-ACTH administration for the female and about $46 \mathrm{~h}$ for the male (Fig. 1), and respective steroid values returned to baseline within 95 - $120 \mathrm{~h}$ postinjection in both individuals. 

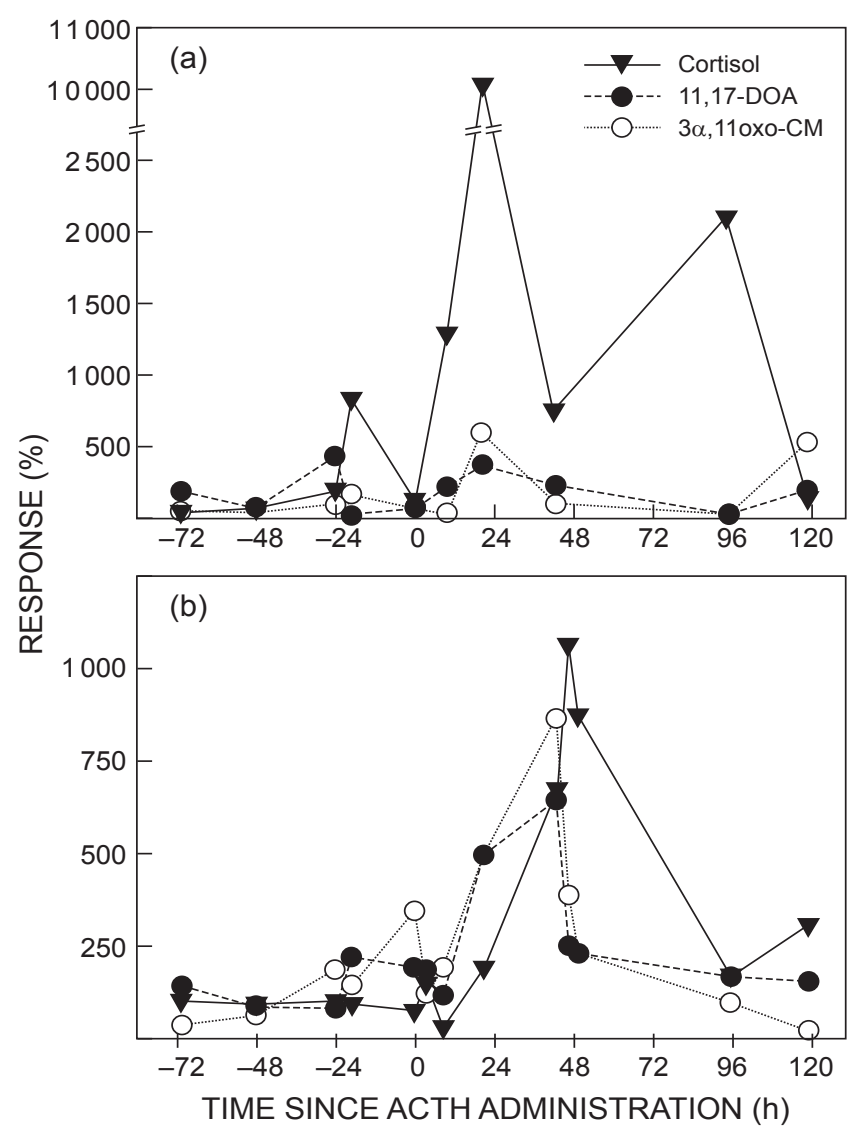

Figure 1: Results from three enzyme immunoassays (cortisol, 3a,110xo-CM and 11,17-DOA) used to measure the immuno-reactive response in bat-eared fox faeces from a female (a) and a male (b) following administration of synthetic adrenocorticotropic hormone. Percentage response indicates strength of response to the introduced hormone, with $100 \%$ response denoting a doubling of fecal glucocorticoid metabolite concentration above baseline values

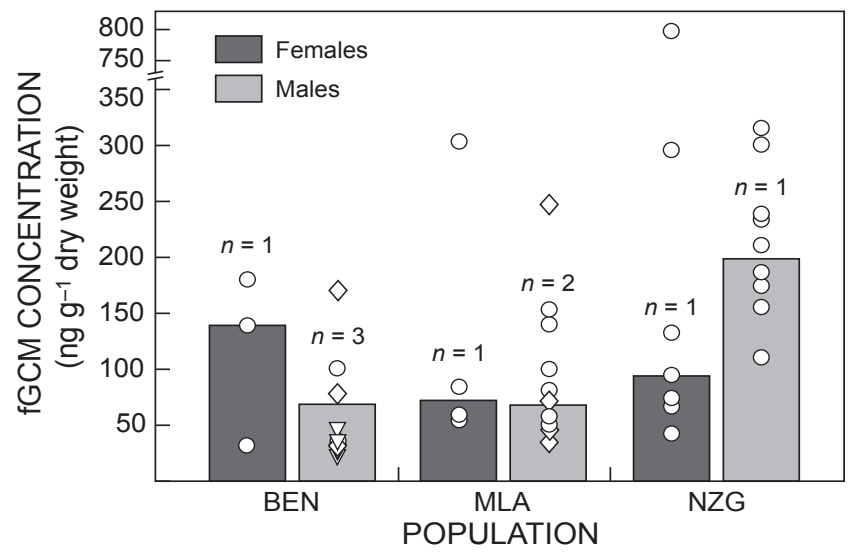

Figure 2: Dot-bar plots of faecal glucocorticoid metabolite (fGCM) concentrations in three bat-eared fox populations (free-ranging: Benfontein Nature Reserve [BEN]; captive: My Little Africa [MLA] and National Zoological Gardens [NZG]). Each symbol represents a value and different types of symbols represent different individuals ( $n=$ number of individuals in each location/sex category). Each bar represents the median fGCM value per site per sex. The presen-tation here reflects low sample sizes and relatively high individual variation, which necessitated non-parametric statistics (see text) 
Mean fGCM concentrations decreased between $13.0 \%$ and $25.9 \%$ in samples frozen 1 - $24 \mathrm{~h}$ post-defecation, with variation in $\mathrm{fGCM}$ concentration between triplet subsamples ranging from 8.1 to $54.6 \%$ for respective measuring points in time.

\section{Comparison of fGCM levels in three populations of bat-eared foxes}

There was no effect of sex on fGCM concentrations in bat-eared foxes (Fig. 2: $\left.X^{2}=0.106, d f=1, P=0.745\right)$, but the three sites differed significantly $\left(X^{2}=\right.$ 16.123, $\mathrm{df}=2, \mathrm{P}<0.001$ ), with $\mathrm{fGCM}$ concentrations in the pair of foxes housed at the NZG being higher than those at MLA and BEN (Fig. 2). Note that only the NZG study subjects' typical schedule and housing conditions were disrupted during the study period, with individuals being separated for five days prior to the ACTH challenge.

\section{Discussion}

Although all three tested EIAs revealed a distinct elevation in fGCM concentrations post ACTH administration, the cortisol EIA emerged as the most suitable assay for determining $\mathrm{fGCM}$ concentrations in wild and captive bat-eared foxes, exhibiting the most distinctive response out of three different assays, and limited fluctuation around baseline fGCM levels. While the serum cortisol measures indicated only a slight increase in circulating cortisol levels, this moderate increase post-ACTH injection might be a result of the delayed collection of the first blood sample (30 minutes post capture). It is likely that the capturing procedure had already instigated a stress response in the test subjects, subsequently reflected in already elevated serum cortisol levels in the first blood sample. Although species-specific differences in steroid metabolism occur in even closely related species (Schwarzenberger 2007) the fGCM values in our study, ranging from an average of 50 to $200 \mathrm{ng} / \mathrm{g}$ dry weight (DW), fell within the expected range of values for canids as determined by similar methods: for example, aardwolves' fGCM range between 300 and 600ng/g DW (Ganswindt, Muilwijk, et al. 2012), maned wolves vary between 32 and 38ng/g DW (Vasconcellos et al. 2011), and African wild dogs have a typically fGCM range from $39-583 \mathrm{ng} / \mathrm{g}$ DW (Monfort et al. 1998). In our 
study, as well as these other investigations, inter-individual and intersexual variation in fGCM levels appears high. However, it is highly likely that the marked intersexual difference suggested by the post-ACTH data was linked to the injury that the female had sustained during the capture procedure (see also Muenscher et al. 2010; Narayan et al. 2013). We found no clear, unidirectional intersexual differences in circulating cortisol levels or fGCM, and therefore predict that larger studies will find healthy male foxes exhibiting cortisol levels comparable to non-lactating female bat-eared foxes.

The similarity in fGCM levels between the captive MLA animals and wild BEN population suggests that captivity does not necessarily lead to excessive stress in socially housed bat-eared foxes. Indeed, this observation is supported by the significantly higher fGCM concentrations found for the two NZG bat-eared foxes, who were temporarily separated from one another in the period directly preceding the ACTH challenge. These foxes' elevated fGCM levels - measured before capture - indicate that they experienced stress or anxiety likely attributable to social separation (Morgan and Tromborg 2007), since their diet and physical location did not change. This agrees with several other studies that indicate mammals' sensitivity to inappropriate social housing, and the impact of isolation on individual health and disease susceptibility (Tamashiro et al. 2005).

We conclude that the cortisol EIA used in this study is a reliable technique for assessing adrenocortical activity in both wild and captive bateared foxes, using faeces as steroid matrix. The fGCM measured using this method appears to be a reliable indicator of stress experienced by the individuals concerned. While the technique is not complicated, we advocate the use of large sample sizes before drawing conclusions or making biologically meaningful inferences, in the light of significant individual variation shown in this study. Future studies will be used to ascertain the influence of lactation on the physiological stress experienced by female bat-eared foxes, while the method can also be used to guide animal husbandry practices in any facility housing captive bat-eared foxes. 


\section{References}

- Clark, H. O., Jr. 2005. Otocyon megalotis. Mammalian Species 766: 15.

- Conforti, V. A., R. G. Morato, A. M. Augusto, L. de Oliveira e Sousa, D. M. de Avila, J. L. Brown, J. J. Reeves. 2012. Noninvasive monitoring of adrenocortical function in captive jaguars (Panthera onca). Zoo Biology 31: 426-441.

- Cook, N. J. 2012. Minimally invasive sampling media and the measurement of corticosteroids as biomarkers of stress in animals. Canadian Journal of Animal Science 92: 227-259.

- Fieß, M., M. Heistermann, J. K. Hodges. 1999. Patterns of urinary and fecal steroid excertion during ovarian cycle and pregnancy in the African elephant (Loxodonta Africana). General and Comparative Endocrinology 115: 76-89.

- Ganswindt, A., J. L. Brown, E. W. Freeman, A. J. Kouba, L. M. Penfold, R. M. Santymire, M. M. Vick, N. Wielebnowski, E. L. Willis, M. R. Milnes. 2012. International Society for Wildlife Endocrinology: the future of endocrine measures for reproductive science, animal welfare and conservation biology. Biology Letters 8: 695-697.

- Ganswindt, A., M. Heistermann, S. Borragan, J. K. Hodges. 2002. Assessment of testicular endocrine function in captive African elephants by measurement of urinary and fecal androgens. Zoo Biology 21: 27-36.

- Ganswindt, A., C. Muilwijk, M. Engelkes, S. Muenscher, H. Bertschinger, M. Paris, R. Palme, E. Z. Cameron, N. C. Bennett, F. Dalerum. 2012. Validation of noninvasive monitoring of adrenocortical endocrine activity in ground-feeding aardwolves (Proteles cristata): exemplifying the influence of consumption of inorganic material for fecal steroid analysis. Physiological and biochemical zoology 85: 194199.

- Ganswindt, A., A. S. W. Tordiffe, E. Stam, M. J. Howitt, F. Jori. 2012. Determining adrenocortical endocrine activity as a measure of stress in 
African buffalo (Syncerus caffer) based on faecal analysis. African Zoology 47: 261-269.

- Goymann, W. 2012. On the use of non-invasive hormone research in uncontrolled, natural environments: the problem with sex, diet, metabolic rate and the individual. Methods in Ecology and Evolution 3: 757-765.

- Hodges, K., J. Brown, M. Heistermann. 2010. Endocrine monitoring of reproduction and stress. In Wild mammals in captivity: Principles and techniques for zoo management, edited by D. G. Kleiman, K. V. Thompson, C. K. Baer. Chicago: University of Chicago Press.

- Hulsman, A., F. Dalerum, A. Ganswindt, S. Muenscher, H. j. Bertschinger, M. Paris. 2011. Non-invasive monitoring of glucocorticoid metabolites in brown hyaena (Hyaena brunnea) feces. Zoo Biology 30: 451-458.

- le Roux, A., Beizhuisen, R., Brekelmans, W., Ganswindt, A., Paris, M., Dalerum, F. 2014. Innovative parental care in a myrmecophageous mammal. Acta Ethologica 17: 63-66.

- Marneweck, S. D., E. Z. Cameron, A. Ganswindt. 2015. Behavioral and endocrine correlates to the aardwolf mating system. Mammalian Biology 80: 31-38.

- $\quad$ Monfort, S. L., K. L. Mashburn, B. A. Brewer, S. R. Creel. 1998. Evaluating adrenal activity in African wild dogs (Lycaon pictus) by fecal corticosteroid analysis. Journal of Zoo and Wildlife Medicine: 129-133.

- Morgan, K. N., C. T. Tromborg. 2007. Sources of stress in captivity. Applied Animal Behaviour Science 102: 262-302.

- Möstl, E., R. Palme. 2002. Hormones as indicators of stress. Domestic Animal Endocrinology 23: 67-74.

- Muenscher, S., M. Henley, R. Palme, P. Thompson, H. Bertschinger. 2010. Concentrations of faecal glucocorticoid metabolites in physically injured free-ranging African elephants (Loxodonta africana). Wildlife Biology 16: 323-332.

- Narayan, E. J., T. Parnell, G. Clark, P. Martin-Vegue, A. Mucci, J.-M. Hero. 2013. Faecal cortisol metabolites in Bengal (Panthera tigris tigris) 
and Sumatran tigers (Panthera tigris sumatrae). General and Comparative Endocrinology 194: 318-325.

- Palme, R., E. Möstl. 1997. Measurement of cortisol metabolites in faeces of sheep as a parameter of cortisol concentration in blood. Zeitschrift fur Saugetierkunde 62 (Suppl 2): 192-197.

- Palme, R., S. Rettenbacher, C. Touma, S. M. El-Bahr, E. Möstl. 2005. Stress hormones in mammals and birds: comparative aspects regarding metabolism, excretion, and noninvasive measurement in fecal samples. Annals of the New York Academy of Sciences 1040: 162-171.

- Pauw, A. 2000. Parental care in a polygynous group of bat-eared foxes, Otocyon megalotis (Carnivora: Canidae). African Zoology 35: 139-145.

- Schwarzenberger, F. 2007. The many uses of non-invasive faecal steroid monitoring in zoo and wildlife species. International Zoo Yearbook 41: 52-74.

- Sheriff, M. J., B. Dantzer, B. Delehanty, R. Palme, R. Boonstra. 2011. Measuring stress in wildlife: techniques for quantifying glucocorticoids. Oecologia 166: 869-887.

- Tamashiro, K. L. K., M. M. N. Nguyen, R. R. Sakai. 2005. Social stress: From rodents to primates. Frontiers in Neuroendocrinology 26: 27-40.

- Touma, C., R. Palme. 2005. Measuring fecal glucocorticoid metabolites in mammals and birds: The importance of validation. Annals of the New York Academy of Sciences 1046: 54-74.

- Vasconcellos, A. S., M.-O. M. Chelini, R. Palme, M. A. B. V. Guimaraes, C. A. Oliveira, C. Ades. 2011. Comparison of two methods for glucocorticoid evaluation in maned wolves. Pesquisa Veterinária Brasileira 31 (Suppl. 1): 79-83. 